\title{
An Arabidopsis thaliana (Ler) inbred line AFDL exhibiting abnormal flower development mainly caused by reduced $A P 1$ expression
}

\author{
QI XiaoLi ${ }^{1,2,3}$, JIANG Yao ${ }^{2}$, TANG Fang ${ }^{2}$, WANG MinJie ${ }^{2}$, HU JianJun ${ }^{2}$, ZHAO ShuTang ${ }^{2}$, \\ SHA Wei ${ }^{1,4} \&$ LU MengZhu ${ }^{2 *}$ \\ ${ }^{1}$ College of Life Sciences, Northeast Forestry University, Harbin 150040, China; \\ ${ }^{2}$ Laboratory of Biotechnology, Research Institute of Forestry, Beijing 100091, China; \\ ${ }^{3}$ College of Life Sciences, Jiamusi University, Jiamusi 154007, China; \\ ${ }^{4}$ Key Laboratory of Genetic Engineering, Qiqihar University, Qiqihar 161006, China
}

Received June 22, 2010; accepted November 10, 2010

\begin{abstract}
The genetic network controlling flowering and flower development consists of a set of floral integrator genes that play a role in light sensing, hormone signaling and developmental pathways. These integrators activate the expression of meristem identity genes LEAFY $(L F Y)$ and APETALAI (API) to initiate the flowering transition. However, how the expression of key genes, such as $A P 1$, responds to diverse signals during flower development remains largely unknown. Here, we report that an Arabidopsis abnormal flower development inbred line (AFDL) exhibits a phenotype similar to the apl mutant, with delayed flowering time and a high frequency of transition of flower meristems into inflorescence meristems after flowering. The flower organs with an abnormal first whorl lack the second whorl and the increased number of inflorescences at the first- and second-whorl positions most closely resembled the phenotypes of apl/cal double mutants. Interestingly, both normal and abnormal flowers coexisted in a single individual. Microarray and quantitative real-time PCR analysis revealed that the expression of $A P 1$ was significantly reduced, while the expression of its interacting genes TERMINAL FLOWER 1 (TFL1), SHORT VEGETATIVE PHASE (SVP), AGAMOUS-like 24 (AGL24), SEPALLATA (SEP) and CAULIFLOWER (CAL) and upstream genes FLOWERING LOCUS C (FLC) and FLM were increased in AFDL, which could serve to explain its phenotype. The expression of genes responsive to different stimuli dramatically changed in AFDL relative to the wild type, as revealed by the differential display of transcripts, indicating that this expression variation is subject to a threshold, leading to an on/off expression pattern of the master regulatory gene (such as $A P 1$ ) of flower development.
\end{abstract}

Arabidopsis thaliana, gene regulation, flower development, AP1, microarray, RT-PCR

Citation: Qi X L, Jiang Y, Tang F, et al. An Arabidopsis thaliana (Ler) inbred line AFDL exhibiting abnormal flower development mainly caused by reduced AP1 expression. Chinese Sci Bull, 2011, 56: 39-47, doi: 10.1007/s11434-010-4263-4

Plants have developed many mechanisms to sense and respond to environmental cues to achieve a successful transition from vegetative to reproductive growth (for a review see [1]). This transition has been intensively studied in Arabidopsis thaliana, in which photoperiod, day length, vernalization, ambient temperature and nutrient status influence this process synergistically [2]. The flowering time genetic network in $A$. thaliana represents one of the

*Corresponding author (email: lumz@caf.ac.cn) best-studied functional systems in plants, and integrates environmental and physiological information to modulate expression of key downstream genes controlling the appropriate time for flowering $[3,4]$. These genes include FLOWERING LOCUS C (FLC), whose expression is regulated mainly by vernalization and an autonomous pathway, and CONSTANS $(\mathrm{CO})$, regulated by an autonomous pathway [5]. These two genes further regulate integrators SUPPRESSOR OF OVEREXPRESSION OF CO 1 (SOC1) and $F T$, which are also regulated by the GA pathway to 
trigger the floral meristem identity genes $L E A F Y(L F Y) / A P 1$ $[6,7]$. LFY and AP1 upregulate each other and play a pivotal role in specifying floral meristems during the conversion of meristem identity from inflorescence to flower under the control of endogenous and environmental cues [8,9].

The floral homeotic gene AP1 specifies floral meristem, sepal and petal identities in Arabidopsis. Flowers of apl plants show a transformation of the first-whorl organs into bract-like structures and the development of flower buds in the axil of each bract-like organ. As a result, these secondary flowers may also produce tertiary and quaternary flowers. Secondary and tertiary flowers can be incomplete or irregular in terms of mosaic organs or possess fewer organs than normal $[10,11]$. In addition to aberrant development of first-whorl organs as bract-like structures, ap 1-1 mutants also lack petals. On secondary and tertiary flower buds, organs occasionally develop mosaics of leaf-like and stamen-like tissue. These organs appear to arise from a region in the meristem where the first- and third-whorl primordia are closely apposed [11,12]. More significantly, in an ap $1 /$ cal double mutant, flower meristems lose their floral fate, leading to a transition to inflorescence architecture and resulting in a complex branched structure [11].

The floral meristem identity gene $A P l$ is essential for emerging floral meristems and flower organ formation by repressing the expression of $S V P, A G L 24$ and SOC1, which promotes inflorescence fate rather than flower formation of the meristem [13]. SOC1, FRUITFUL (FUL) and AGL24 play roles in activation of $L F Y / A P 1$ during the emergence of flower meristems at the side of SAM, thus forming a feedback regulation loop to regulate spatial patterning precisely in the SAM upon floral transition and the ultimate organization of inflorescence architecture [1]. API and its homologous gene $C A L$ are activated in the stage 1 floral meristem following the domain expressing $L F Y[14,15]$. Although LFY binds in vivo to $A P l$ and $C A L$ regulatory sequences, it alone is insufficient to activate $A P 1[16,17]$. FT was shown to induce $A P 1$ expression [18], although the underlying mechanism still remains to be elucidated. APl transcripts are also negatively regulated by the AP3/PI heterodimer in combination with other unknown factors to restrict its spatial distribution during early stages of floral development [19]. API also interacts with $S E P 1 / 2 / 3 / 4$, which are MADSbox genes playing roles in the ABCE model of flower development, to specify floral meristem identity and organ identity [20-22]. Recently, it was found that PUCHI and $B O P$ are required for specification of floral meristem identity by providing a positional cue for $L F Y$ and $A P l$ to be expressed when induced by flower-promoting signals such as photoperiod [23]. The authors also suggested that negative factor(s), such as TFL1, which is known to limit $L F Y$ expression to the floral meristem, may repress PUCHI function in the secondary inflorescence meristem. Therefore, the regulation of $A P I$ by endogenous and environmental cues warrants further investigation.
Existing evidence points to AP1 having functions for both onset of flowering and first- and second-flower organ development. It can interact with many genes, such as $C A L$, FUL/AGL8, LFY, AG, FT, TFL1, SVP, AGL24, SOC1 and $A P 3 / P I$, which were discovered by forward genetic screening of Arabidopsis mutants. Further investigation is therefore needed to understand the regulation of $A P 1$, particularly in response to endogenous and environmental cues. However, a mutagenesis approach limits the ability to examine crosstalk among so many genes that participate in a variety of light-sensing, hormone-signaling and developmental pathways [24]. In this paper, we describe an abnormal flower development line (AFDL) that exhibits a similar, but not identical, flower phenotype to the apl mutant. This phenotype was primarily caused by significant downregulation of AP1 expression in conjunction with other genes that regulate flowering and flower development. Phenotypic and genetic analysis of AFDL revealed that the regulation of $A P 1$ and its interaction with other flowering regulators are complicated and sensitive to both internal and external stimuli.

\section{Materials and methods}

\subsection{Plant materials}

Arabidopsis thaliana Landsberg erecta (Ler) seeds were obtained from the Nottingham Arabidopsis Stock Centre (NASC). We found a transposon (Ds) insertional mutant (At1g52910) exhibiting abnormal flower development (thus named AFDL) when screening Arabidopsis mutants associated with the development of vascular tissues. Homo- and heterozygosity identification of the mutated gene was performed on AFDL and the results showed that an insertional mutation of the At1g52910 gene was inconsistent with the abnormal phenotype, indicating that the phenotypes of AFDL were not caused by mutation of the At1g52910 gene. RNAi and complementation constructs for this gene were then introduced into wild-type (WT) and AFDL lines, respectively, but the AFDL phenotypes did not emerge in RNAi transgenic plants and the WT phenotype was not observed in transgenic AFDL plants. This provided further evidence that the phenotype of AFDL does not result from mutation of the At1g52910 gene. Furthermore, the result of Tail-PCR analysis did not reveal any other $D s$ insertion sites, suggesting that AFDL is not caused by a $D s$ insertional mutation. In addition, we analyzed segregation in one selfing AFDL plant and found that it was not caused by a single nuclear mutation because the ratio of plants with abnormal and normal phenotypes deviated from Mendel's law. Finally, the phenotype of AFDL was mostly stable after multigeneration selfing, pointing to a genetic basis.

AFDL and WT plants were vernalized at $4{ }^{\circ} \mathrm{C}$ for $2 \mathrm{~d}$ and grown on a mixture of turf soil and vermiculite $(3: 1)$ at $(22$ $\pm 2)^{\circ} \mathrm{C}$ in short days (SD: $8 \mathrm{~h}$ light, $16 \mathrm{~h}$ dark) and long days 
(LD: $16 \mathrm{~h}$ light, $8 \mathrm{~h}$ dark), to check flowering time and for phenotypic observation. Plants were also grown on MS media in petri dishes $(120 \mathrm{~mm})$ at $(22 \pm 1)^{\circ} \mathrm{C}$ under LD conditions for microarray analysis.

\subsection{Phenotypic observation}

Intact plants were visually checked and photographed. The flowering time was measured by counting the number of leaves at bolting with $0.5-1.0 \mathrm{~cm}$ stem above the rosette. The data were statistically analyzed using one-way ANOVA analysis of variance and least significant difference tests with SPSS 15.0 software (SPSS, Inc., Chicago, IL, USA). Flower buds were observed with a Zeiss SteREO Discovery V8 stereomicroscope and KYKY-2800B scanning electron microscope (KYKY Technology Development, Beijing, China). Specimens were prepared using the t-butyl alcohol freeze-drying method for scanning electron microscopy (SEM) [25]. Images were documented with software installed on the instruments.

\subsection{Genomic sequencing of $A P 1$ and its upstream region}

Based on the genomic sequence of the APl region (TAIR: http://www.arabidopsis.org), we designed primers (Table $\mathrm{S} 1)$ to span the whole region of the $A P l$ genomic sequence to the end of the upstream adjacent gene. Amplified DNA fragments from both AFDL and WT lines were used for direct sequencing. The sequence data were assembled and compared between AFDL and WT.

\subsection{RNA isolation and labeling}

To identify differences in gene expression between AFDL and WT, we used a bulked sampling strategy to minimize individual variation, by collecting and mixing seedlings with six clear rosette leaves in petri dishes and emerging flower buds at bolting from more than 50 plants of both AFDL and WT. The analysis was combined with the differential display method using Affymetrix Arabidopsis ATH1 GeneChips (Affymetrix, Santa Clara, CA, USA). These bulked samples and cauline leaves (for quantitative real-time PCR (qRT-PCR)) were frozen in liquid nitrogen and stored at $-80^{\circ} \mathrm{C}$. Total RNA isolation was performed with the Plant RNeasy Mini Kit (Qiagen China, Shanghai) following the manufacturer's instructions. Five micrograms of total RNA was used to synthesize double-stranded cDNA using the Affymetrix One-cycle cDNA Synthesis Kit. The cDNAs acquired were used as a template for synthesis of biotinylated cRNA using the GeneChip IVT Labeling Kit (Affymetrix). Biotinylated cRNA was purified with the Affymetrix GeneChip Sample Cleanup Module and fragmented according to the manufacturer's protocol (Affymetrix).

\subsection{Array hybridization and analysis of expression data}

Hybridization of GeneChip arrays was done in an Affymetrix Hybridization Oven 640 following the manufacturer's protocol (Affymetrix). The Affymetrix Fluidics Station 450 was used to facilitate washing and staining. Chips were scanned by a GeneChip ${ }^{\circledR}$ Scanner 3000 7G. Expression levels in seedlings and flower buds were calculated from Affymetrix hybridization intensity data and differences between AFDL and WT were compared using preinstalled GeneChip Operating Software.

\subsection{Quantitative real-time PCR}

Reverse transcription was performed using the ThermoScript RT-PCR System (Invitrogen) according to the manufacturer's instructions, starting with $3 \mu \mathrm{g}$ of the total RNA isolated from flower buds and cauline leaves. Primers specific to the flower-related genes $A P 1, A P 2, A P 3, S E P 1$, SEP2, SEP3, SEP4, FUL, CAL, LFY, AGL24, SOC1, SVP, SAP18, FLC, FD, FT, TFL1, CO, FLM, SVP and TUBULIN were used for qRT-PCR (Tabke S2). Amplification was monitored in real time with an ABI 7500 Real-Time PCR System (Applied Biosystems, Shanghai, China) using SYBR Green PCR Master Mix (Applied Biosystems) as the fluorescence source. The relative expression levels were calculated and statistically analyzed using SDS software v1.4 (Applied Biosystems).

\section{Results}

\subsection{Phenotypic variation of AFDL}

AFDL plants grew vigorously but exhibited variable degrees of phenotypic alteration compared to WT (Figure 1(a)) as follows: (i) more inflorescences stacked at a small angle to the stem (Figure 1(b)); (ii) long, thin inflorescences with a larger angle to the stem and longer internodes (Figure 1(c)); and (iii) infrequent coexistence of both WT and AFDL inflorescence phenotypes (Figure 1(d)-(f)) as well as the presence of both normal (Figure $1(\mathrm{~g})$ ) and abnormal flowers (Figure 1(h)) in the same plant (Figure 1(f)). All of these plants produced more inflorescence branches with cauline leaves because of the conversion of the first-whorl organ into a 2nd inflorescence, and 3rd inflorescences emerged from the first-whorl organ on the secondary inflorescence, until seven or more inflorescences were produced. Multiple flowers towards the axillary side showed a cauliflower-like shape, with no petals being observed in AFDL types (Figure $1(\mathrm{~h})$ ), which is very similar to apl/cal double mutants. Compared to the WT line, no obvious phenotypic alteration or growth rate changes of AFDL plants were observed in the vegetative phase starting from young seedlings to adult plants that had not yet bolted (Figure 2(a)-(f)). 


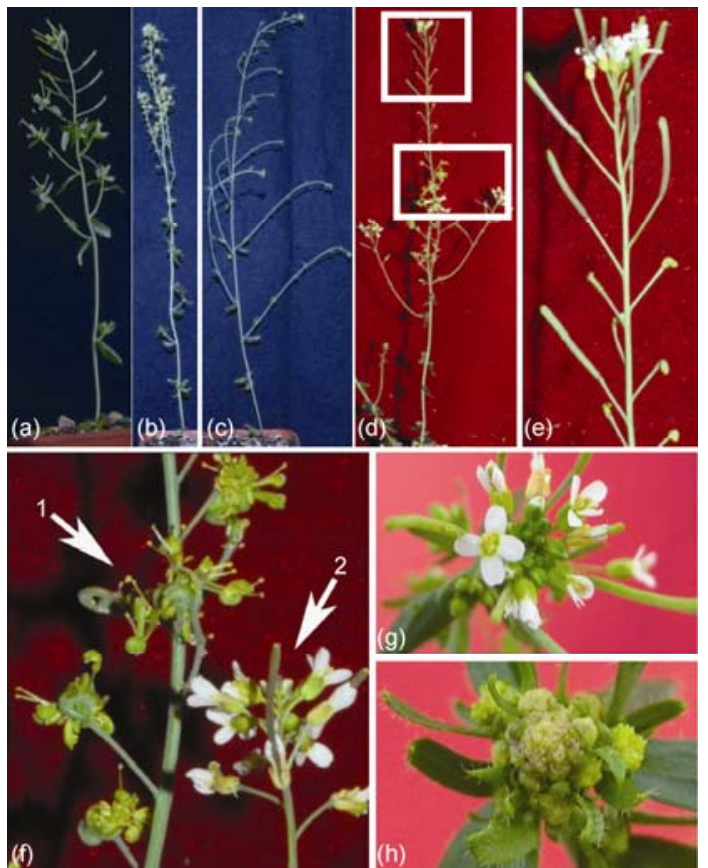

Figure 1 Phenotype of WT (a, g) and AFDL (b-f, h) lines. Inset boxes in (d) indicate parts that have been magnified in (e) and (f). Arrows 1 and 2 indicate an abnormal and normal flower, respectively.

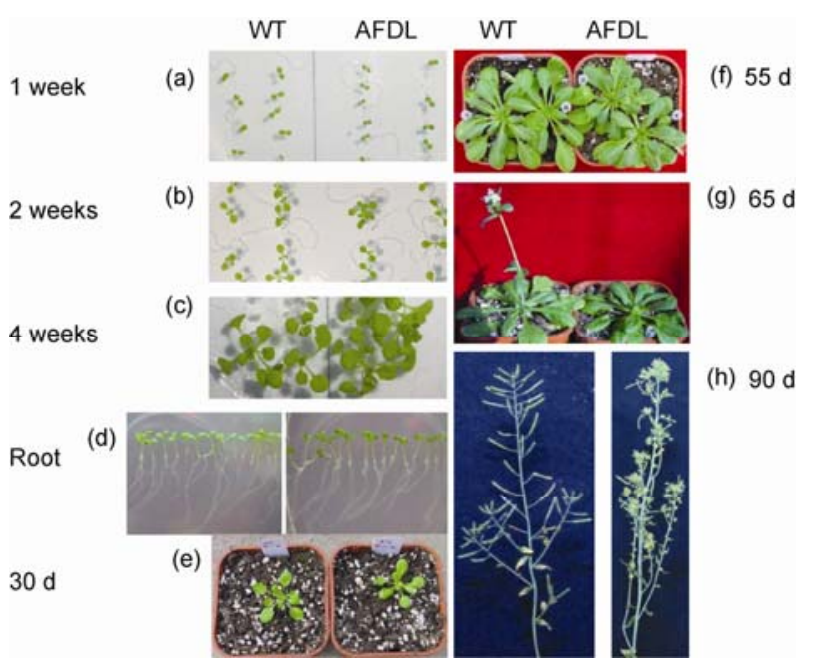

Figure 2 Development of AFDL and WT plants. (a)-(d) Plants growing in MS medium; (e)-(h) plants growing in soil.

However, delayed bolting and flowering were observed in AFDL plants (Figure $2(\mathrm{~g})$ and $(\mathrm{h}))$, and significant $(P<$ $0.05)$ differences in leaf number $(\mathrm{LN})$ at bolting $(0.5-1.0 \mathrm{~cm}$ above the rosette) were observed between AFDL and WT plants of approximately 10 in the SD condition and 3 in the LD condition (Figure 3). Therefore, AFDL is a late-flowering type under both SD and LD conditions.

The flowers of AFDL plants exhibited similar phenotypes (Figure 4(b)) to the apl-1 mutant [11]. However, greater variation was observed in AFDL flowers. (i) The first-whorl organ was either normal, absent or converted to a bract-like, carpelloid, and stamenoid sepal (Figure 4(b),

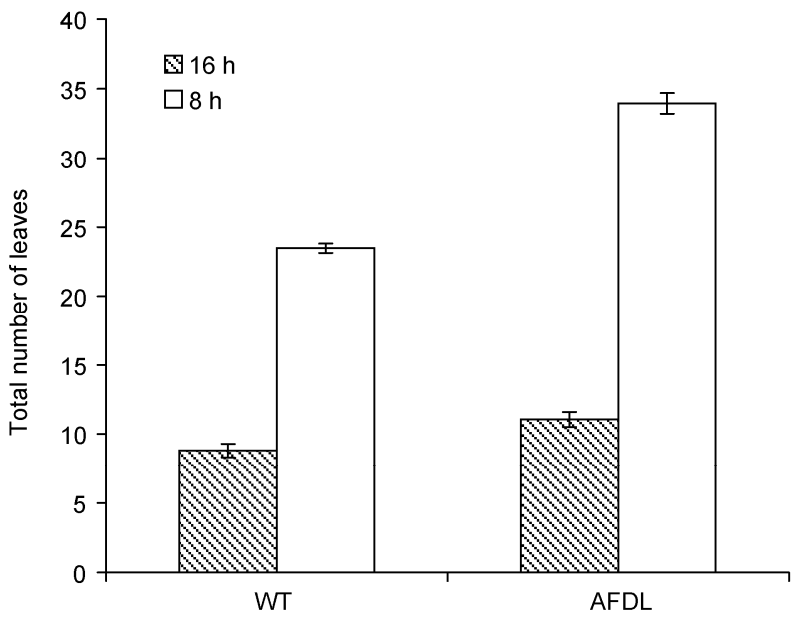

Figure 3 Flowering time in AFDL plants. Flowering time is represented by the number of rosette leaves formed prior to bolting. Filled and empty bars indicate plants grown under long- and short-day conditions, respectively. Error bars represent the standard deviation.

(e)-(i)) or other mosaic organs (Figure 4(f) and (g)). Secondary flowers (Figure 4(b) and (c)) or inflorescences (Figure $4(\mathrm{~d})$ ) arose in the axils of leaf-like organs. (ii) The 2nd-whorl organ was commonly absent in AFDL (Figure 4(b), (g)-(i)) or incompletely homeotically converted to a sepalloid petal (Figure 4(j)), and occasionally some anther tissue was present on the top of the petal (Figure 4(k)). (iii) A reduced number of stamens and green filaments were often observed at 3rd-whorl positions (Figure 4(h) and (j)). Some stamens had a chimeric structure with green filament stamens and stigmatic papillae on the distal tip of the anther (Figure 4(h)). (iv) An opened carpel, sometimes with anthers at its margin (Figure 4(n)), appeared in 4th-whorl positions. Sometimes a carpel fused with a stamen was also observed (Figure 4(l) and (m)). An extreme phenotype was observed in which more complex structures such as stamens (Figure 4(o) and (p)) appeared in the carpel. This phenotypic variation was most similar to that of agl24/svp double and agl24/svp/apl triple mutants [26]. In addition to variation in the 4 whorls, chimeric structures often appeared on inflorescences (Figure 4(q)-(t)). Compared to WT plants (Figure 4(u)), normal (Figure 4(v)) and twisted (Figure $4(w)$ ) siliques and siliques with anther or filament structures (Figure 4(x)) were also observed in AFDL. We also used SEM to document flower bud development. Similar to a strong apl mutant, first-whorl organs in AFDL plants appeared in an order similar to that of WT sepals (Figure 4(y)) but did not grow to enclose the developing flower bud (Figure 4(aa)). The inflorescence primordia were more rounded, growing away from the central floral meristems (Figure $4(z)$ ), and were present in the axils of first-whorl organs, leading to crowded flower buds (Figure 4(aa)).

\subsection{Microarray analysis of AFDL}

No differences in the genomic sequence of the $A P I$ region, 


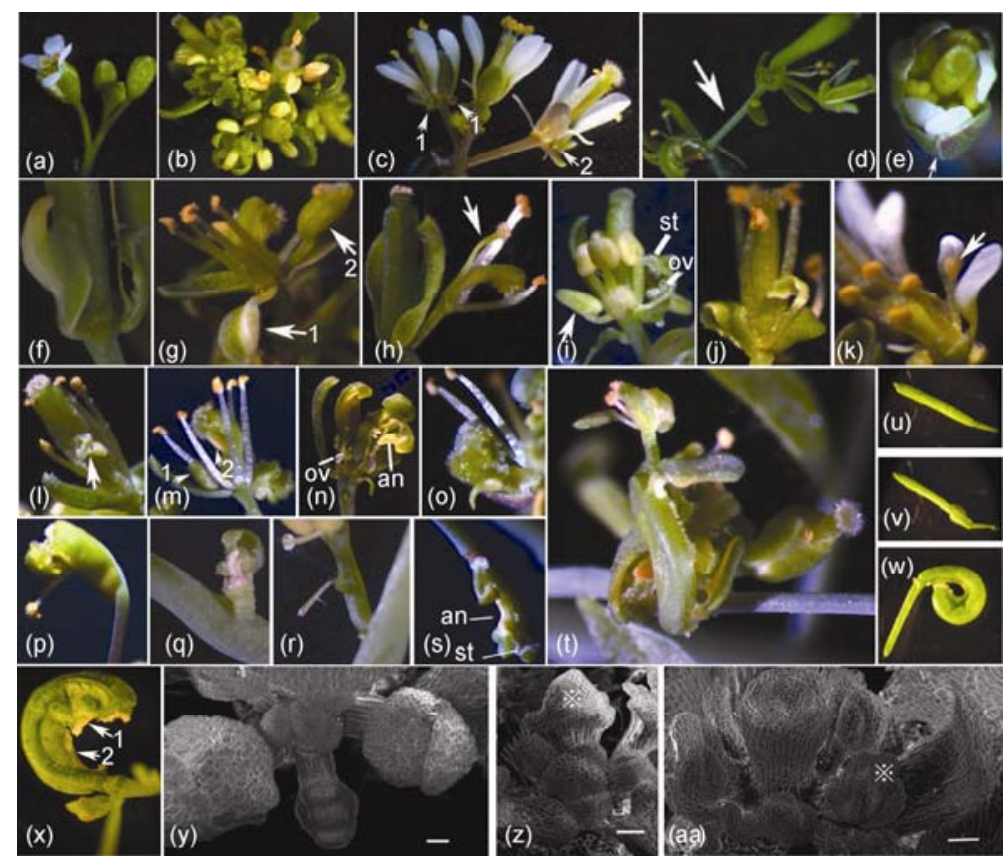

Figure 4 Phenotypic variation in flowers and siliques of AFDL plants. (a), (u) and (y) WT; (b)-(aa) AFDL. (a) Normal flowers; (b) AFDL flowers; (c) secondary flowers formed at the positions of a first whorl (arrow 1) or in the axils of leaf-like organs (arrow 2) of a flower; (d) secondary inflorescence formed in the axils of a first whorl; (e) flower with a fused sepal (arrow) and 3 petals, appearing asymmetric; (f) one sepal is mosaic, the other sepal shows carpelloid features; (g) a mosaic sepal (arrow 1) and an extra pistil with a filament at the base of the pistil (arrow 2); (h) a flower possessing few organs and a chimeric structure, with green filaments and stigmatic papillae on the distal tip of the anther (arrow); (i) a staminoid sepal (arrow), and a sepal with carpelloid features such as stigmatic papillae (st) on the top and multiple ovules (ov) along the margin; (j) sepalloid petals; (k) a flower with staminoid tissue on top of the petals (arrow); (1) a carpel is fused with a stamen (arrow); (m) an extra pistil (arrow 1) and a fused carpel with a stamen (arrow 2); (n) flower with a sepal and three carpels, with anther tissue (an) developed on the top margin of these unfused carpels; (o) an unfused carpel with a chimeric structure resembling a stamen; (p) a partly fused carpel with a stamen; (q) a chimeric structure on a stem; (r) a chimeric structure on a pedicel; (s) a carpel-like structure standing on a top of pedicel; (t) a chimeric structure with inflorescence on carpel-like tissue; (u) silique of WT; (v) a normal silique of AFDL; (w) a twisted silique of AFDL; (x) anther-like (arrow 1) and filament-like tissues (arrow 2) at the side of an AFDL silique; (y) floral buds of WT under SEM. (z) and (aa) Floral buds of AFDL under SEM. (z) A higher order of inflorescence meristem (asterisk) appearing at the side of a developing flower bud; (aa) an inflorescence meristem (asterisk) emerging in the axil of the first-whorl organ. An, anther; ov, ovules; st, stigmatic papillae; tr, trichomes. Bar $=20 \mu \mathrm{m}$.

including $A P 1$ and its $5^{\prime}$ regulatory sequences were found between AFDL and WT strains (Figure S1). To find out which gene(s) could be responsible for the altered flower development in AFDL, we employed a differential display of transcripts in bulked samples of seedlings prior to bolting and flower buds upon bolting, using Affymetrix Arabidopsis whole genome chips, to analyze gene expression. Comparison of the expression data revealed that $95 \%$ of the genes showed no significant difference between AFDL and WT (less than 2-fold). Totals of 1274 and 942 genes were differentially expressed (more than 2-fold) in flower buds (Table S3) and seedlings (Table S4), respectively.

We selected the differentially expressed genes involved in the genetic network regulating flowering and flower development (Table 1), and found that API expression was significantly reduced in the flowers of the AFDL strain, whereas $A P 1$ expression was 128 -fold higher in WT. Flowering time and organ development regulators, such as $F L C$, FT, AGL24, FUL, LFY, SEP4, SVP and SAP (Table 1), and several other less-studied MADS-box homeotic genes, such as AGL8, AGL13, AGL42, AGL44, AGL71 and AGL87, were also differentially expressed (Table S3). We also classified these genes using DAVID software [27,28] according to their biological functions; the gene ontology terms and the number of genes for each term are presented in Table S5. A large group of genes responsive to a variety of stimuli (Table 2) were differentially expressed (Table S6), accounting for $20.3 \%$ and $9.2 \%$ of the total number of differentially expressed genes in seedlings and flower buds, respectively. These are found in much higher proportions compared to the other terms (Table S5). These genes include JAZ1, ARR7, GI and $R G L 2$, which are involved in flowering regulation in response to hormone signaling, and DREBIA, which has been implicated in cold-inducible transcriptional regulation.

\subsection{Gene expression using qRT-PCR}

We further analyzed expression of selected genes both upstream and downstream of $A P 1$ in the network in cauline leaves and flower buds using qRT-PCR (Figure S2). In cauline leaves, both flower-promoting $(F T, F D$ and $C O)$ and flower-inhibiting (FLC, FLM and $S V P$ ) genes were highly expressed (Figure 5(a)), but they were expressed at normal or moderate levels in flower buds (Figure 5(b)). Interestingly, putative downstream genes from $A P 1$, such as $S E P 1$, $S E P 2, S E P 3$ and SEP4, were highly expressed in AFDL in 
Table 1 Expression of genes involved in flowering and flower development

\begin{tabular}{|c|c|c|c|c|}
\hline Probe set ID & Name & Gene ID & Description & Signal (log ratio) \\
\hline 259372_at & $A P 1$ & At1g69120 & Specifies floral meristem and sepal identity. & -6.9 \\
\hline 257220_at & ATMYB21 & At3g27812 & $\begin{array}{l}\text { Encodes a member of the R2R3-MYB transcription factor gene family. Induced by } \\
\text { jasmonate. Involved in jasmonate response during stamen development. }\end{array}$ & -5.2 \\
\hline 256788_at & CYP90D1 & At3g13730 & Encodes a cytochrome P-450 gene involved in brassinosteroid biosynthesis. & -3.3 \\
\hline 249349_at & MYB24 & At5g40350 & $\begin{array}{l}\text { Myb24 transcription factor. Involved in jasmonate response during stamen develop- } \\
\text { ment. }\end{array}$ & -2.2 \\
\hline 264638_at & $F T$ & At1g65480 & $F T$, together with $L F Y$, promotes flowering. & -2.1 \\
\hline 258237_at & $A T M Y B 21$ & At3g27810 & $\begin{array}{l}\text { Encodes a member of the R2R3-MYB transcription factor gene family. Induced by } \\
\text { jasmonate. Involved in jasmonate response during stamen development. }\end{array}$ & -1.8 \\
\hline 246216_at & RОT3 & At4g36380 & Member of the CYP90C CYP450 family. & -1.5 \\
\hline 247276_at & HEN4 & At5g64390 & $\begin{array}{l}\text { Encodes a K homology }(\mathrm{KH}) \text { domain-containing protein. HEN4 acts redundantly with } \\
\text { HUA1 and HUA2 in specification of floral organ identity in the third whorl. }\end{array}$ & -1.5 \\
\hline 265058_s_at & $M B P 2$ & At1g52040 & Encodes myrosinase-binding protein expressed in flowers. & -1.5 \\
\hline 248371_at & GA20OX2 & At5g51810 & Encodes gibberellin 20-oxidase. & -1.4 \\
\hline 247898_at & ZTL & At5g57360 & Encodes clock-associated PAS protein ZTL. & -1.3 \\
\hline 256017_at & $J A Z 1$ & At1g19180 & JAZ1 is a nuclear-localized protein involved in jasmonate signaling. & -1.3 \\
\hline 252780_at & ATA1 & At3g42960 & Arabidopsis homolog of TASSELSEED2. Expressed specifically in tapetal cells. & -1.2 \\
\hline 265542_at & $S Y D$ & At2g 28290 & Encodes a SWI2/SNF2-like protein of the SNF2 subclass. & -1.1 \\
\hline 251635_at & $A D P G 1$ & At3g57510 & Encodes ADPG1, a polygalacturonase protein involved in silique and anther dehiscence. & -1 \\
\hline 258842_at & CLPS3 & At3g04680 & Encodes a nuclear protein that functions in mRNA processing. & -1 \\
\hline 247553_at & FUL & At5g60910 & MADS-box gene negatively regulated by APETALA1. & 1 \\
\hline 254130_at & $A G L 24$ & At4g24540 & Encodes a MADS-box protein involved in flowering. & 1 \\
\hline 264041_at & SEP4 & At2g03710 & $\begin{array}{l}\text { This gene belongs to the family of SEP genes. It is involved in development of sepals, } \\
\text { petals, stamens and carpels. Additionally, it plays a central role in determination of } \\
\text { flower meristem and organ identity. }\end{array}$ & 1 \\
\hline 264054_at & $S V P$ & At2g22550 & $\begin{array}{l}\text { Encodes a nuclear protein that acts as a floral repressor and functions within the ther- } \\
\text { mosensory pathway. }\end{array}$ & 1 \\
\hline 247490_at & $L F Y$ & At5g61850 & $\begin{array}{l}\text { Encodes a transcriptional regulator that promotes the transition to flowering. Involved in } \\
\text { floral meristem development. }\end{array}$ & 1.1 \\
\hline 250476_at & FLC & At5g10140 & $\begin{array}{l}\text { MADS-box protein encoded by FLOWERING LOCUS C-transcription factor that } \\
\text { functions as a repressor of floral transition and contributes to temperature compensation } \\
\text { of the circadian clock. }\end{array}$ & 1.1 \\
\hline 254065_at & GA20OX1 & At $4 \mathrm{~g} 25420$ & $\begin{array}{l}\text { Encodes gibberellin 20-oxidase that is involved in the later steps of the gibberellin bio- } \\
\text { synthetic pathway. Regulated by a circadian clock. Weak expression response to far-red } \\
\text { light. }\end{array}$ & 1.2 \\
\hline 257904_at & $T D F 1$ & At3g28470 & Member of the R2R3 factor gene family. & 1.4 \\
\hline 249716_at & SAP & At5g35770 & $\begin{array}{l}\text { A recessive mutation in the Arabidopsis STERILE APETALA (SAP) causes severe } \\
\text { aberrations in inflorescence and flower and ovule development. }\end{array}$ & 1.5 \\
\hline 259252_at & IBMI & At3g07610 & IBM1 likely encodes a protein with histone $\mathrm{H} 3 \mathrm{mK} 9$ demethylation activity. & 1.5 \\
\hline 252957_at & GRP2 & At4g38680 & Encodes a glycine-rich protein that binds nucleic acids and promotes DNA melting. & 2.8 \\
\hline
\end{tabular}

cauline leaves (Figure 5(a)). Notably, CAL and TFL1 were highly expressed in flower buds (Figure 5(b)) and $A P 1$ expression was confirmed to be significantly reduced (400-500 fold) in AFDL flower buds by qRT-PCR.

\section{Discussion}

We identified an Arabidopsis line, AFDL, showing a phenotype similar to apl, as evidenced by the conversion of first-whorl organ primordia into floral meristems, homeotic changes of the first and second whorls of floral organs, the absence of petals and the conversion of the first sepal whorl into leaf-like structures [11]. However, AFDL flowers exhibit obvious differences when compared to apl mutants. For instance, a much higher frequency of conversion of the floral meristem into an inflorescence meristem is observed, similar to the phenotype of apl/cal double mutants [11], and alterations of the 4th floral whorl (Figure 4(1)-(p)) most similar to that of agl24/svp double and agl24/svp/apl triple mutants [26]. In addition, considerable phenotypic variation was found within AFDL, ranging from weakly to severely altered phenotypes. In extreme cases, both normal and abnormal flowers coexist in the same plant (Figure 1(d)). These results suggest that the genetic control of the AFDL phenotype is complex and deserves further characterization.

The apl-like phenotype of AFDL could be caused by mutation of $A P 1$. Therefore we examined the genomic 
Table 2 Number of differently expressed genes responsive to stimuli in seedlings and flower buds of AFDL plants

\begin{tabular}{lrc}
\hline \multicolumn{1}{c}{ Term } & Count & Percent (\%) \\
\hline Seedling & 107 & 11.5 \\
response to abiotic stimulus & 63 & 6.8 \\
response to organic substances & 57 & 6.1 \\
response to endogenous stimulus & 51 & 5.5 \\
response to hormone stimulus & 47 & 5.1 \\
response to temperature stimulus & 45 & 4.8 \\
response to radiation & 44 & 4.7 \\
response to light stimulus & 42 & 4.5 \\
response to inorganic substances & 35 & 3.8 \\
response to osmotic stress & 32 & 3.4 \\
response to oxidative stress & 32 & 3.4 \\
cellular response to stress & 31 & 3.3 \\
response to salt stress & 26 & 2.8 \\
response to metal ions & 25 & 2.7 \\
response to cold & 24 & 2.6 \\
response to abscisic acid stimulus & 21 & 2.3 \\
response to heat & 21 & 2.3 \\
immune response & 20 & 2.2 \\
innate immune response & 19 & 2.0 \\
response to reactive oxygen species & & \\
Flower buds & 71 & 5.8 \\
response to organic substances & 59 & 4.8 \\
response to endogenous stimulus & 29 & 2.4 \\
response to light stimulus & &
\end{tabular}
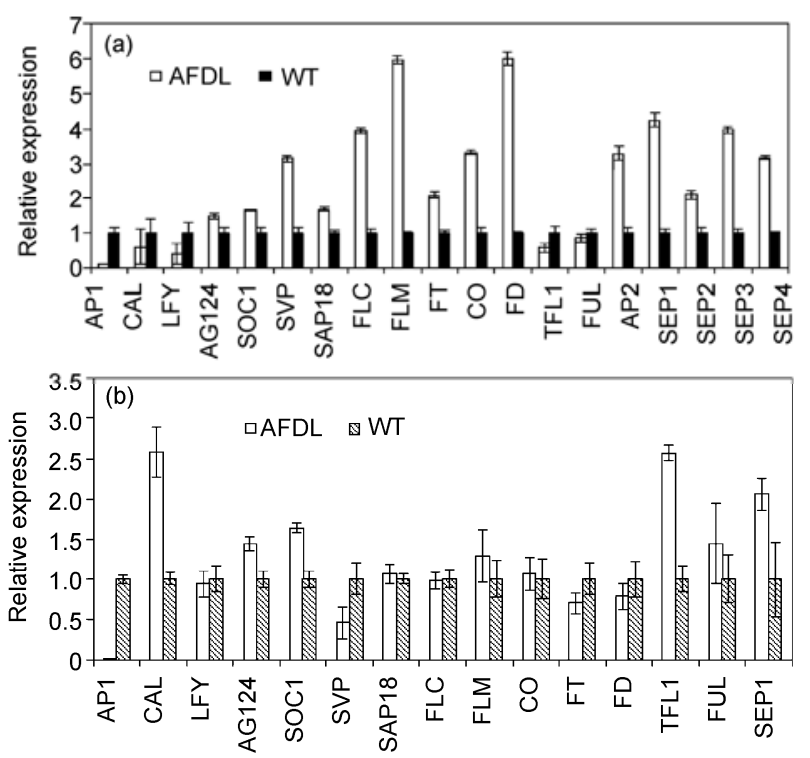

Figure 5 Expression of flowering regulatory genes in cauline leaves (a) and flower buds (b) using qRT-PCR.

sequence of $A P 1$ and its upstream region but failed to find changes in AFDL relative to WT, suggesting that the phenotype cannot be attributed to genomic mutations in this region. The quantitative flower trait of AFDL suggests that the apl-like phenotype of AFDL may be caused by polygenic interactions, particularly given the fact that both typical AFDL and WT inflorescences coexist in one individual.
Therefore, we adopted a 'bulk sampling strategy' (see Materials and methods) in combination with Affymetrix Arabidopsis ATH1 GeneChips to identify genes expressed differentially in seedlings and flower buds between AFDL and WT. Not surprisingly, API was found to be significantly reduced in flowers of AFDL using both microarray (Table 1) and qRT-PCR (Figure 5(b)) data, indicating that there is almost no expression of APl in AFDL. Such a change may explain the apl-like flower of AFDL but is insufficient to explain the delay in flowering, more frequent mosaic flowers and inflorescences in AFDL. The unique phenotype of AFDL flowers most closely resembles the combined phenotypes in mutants for AP1, TFL1, CAL, SVP, $A G L 24, S E P, S A P$ or their compound mutants, suggesting that the phenotypic variation may result from crosstalk among these genes to control flower development. Indeed, AP1, TFL1, CAL, SVP, AGL24, SEP1, SEP4 and SAP were among the genes found to be differentially expressed by microarray (Table 1) and qRT-PCR (Figure 5(b)) in this study. The roles for these genes in flower development have been well characterized, thus implicating their synthetic contributions to development of the AFDL phenotype. In flower buds of AFDL where emerging inflorescence meristems were formed, TFL1 was found to be highly expressed, and this gene can negatively regulate $A P 1$ [29] and specify inflorescence meristem identity [30], leading to a delay of floral meristem formation. Downregulation of $A P I$ can also contribute to the higher levels of SVP in cauline leaves, which negatively regulates expression of the $A P I$ activator FT through direct binding to CArG motifs in the FT sequence [8], and correspondingly results in lower levels of FT and AP1 in flower buds. On the other hand, the low levels of AP1 in flower buds cannot repress expression of $A G L 24$ and $S O C 1$, which promote inflorescence fates rather than flower formation in the meristem [13] and result in more abundant and longer inflorescences in AFDL. Clearly, further analysis of their expression patterns in AFDL is required to elucidate the interactions among these genes.

Furthermore, many cauline leaves were observed in AFDL on higher-order inflorescences that emerged at places where normal flowers would develop in WT. To investigate if they also play a role in regulating flowering time and development, we also analyzed genes that interact with $A P I$ in the network in cauline leaves using qRT-PCR, and found that expression of FLC, FLM, SVP, FT, FD, PI, AP2 and SEPI-4 were highly increased (Figure 5(a)). Therefore, the delay of flowering in AFDL can be explained by high expression of FLC (also in seedlings) and FLM in cauline leaves. These two late flowering regulators are well documented to be responsible for the delayed transition from the vegetative to reproductive phase [31]. However, flower-promoting genes FT and FD were also highly expressed in cauline leaves, while the opposite case was observed in flower buds (Figure 5). The elevated expression of these antagonistic regulators in AFDL cauline leaves warrants 
further investigation, although we can speculate that FT or its transcripts are produced in cauline leaves but somehow fail to be transported to the emerging flower meristem.

Interactions among the aforementioned genes in contributing to the AFDL-specific phenotype may occur in response to sensed endogenous signals and environmental cues, which is evidenced by the finding that $20.3 \%$ of the total number of differentially expressed genes belong to those responding to 36 categories of endogenous signals and stress-related stimuli (Table S6). In addition, both AFDL and WT flowers coexisting in one individual indicates that this interaction is sensitive to slight changes in endogenous and environmental signals. In fact, previous studies have shown the expression of key flowering-time genes to be regulated by different cues. TFL1 plays an important role in establishment and maintenance of the inflorescence meristem [29] and its expression has been shown to respond to changes in sucrose levels [32]. The downregulation of the starch synthase gene (At1g32900, see Supporting Information, also for the following genes) and glucose metabolism-related genes (At3g21370, At1g06000 and At1g19940) but upregulation of the sugar transporter (At4g36670) in AFDL might change the sugar level in the seedling, thus affecting the flowering time. SVP-mediated control of FT gene expression may represent a mechanism used by plants to adjust the timing of flower development under fluctuating temperature conditions [8]. The circadian clock proteins LHY and CCA1 may reduce the abundance of SVP to accelerate flowering by activating FT expression under long days [33]. The reduced transcription of both LHY (At1g01060) and CCA1 (At2g46830), thus leading to the increased SVP (At2g22550) transcription in AFDL, may suggest the involvement of a circadian clock pathway in the expression of its flowering phenotype. The GA pathway may also contribute to the control of flowering in AFDL, because GI (At1g22770, integrating signals from the GA pathway) and RGL2 (At3g03450, major repressor of GA responses) and the genes responsive to other hormones such as JAZ1 (At1g19180, a jasmonate signaling repressor), CRFs (At4g23750 and At3g61630, mediating the transcriptional response to cytokinin) and $A R R 7$ (At1g19050, involved in the negative feedback loop for cytokinin signaling) [34-36] were differentially expressed in AFDL compared to that in WT. In addition, high expression of the cold-inducible transcription regulator DREB1A (At4g25480) also hints at crosstalk between cold-inducible and flowering pathways, which is another fine-tuning mechanism for control of flowering time [37]. This situation strongly resembles the recent finding that phenotype is a consequence of large variations in gene expression that are thresholded during development to determine cell fate in Caenorhabditis elegans [38].

Discrepancies between phenotypes of apl and AFDL may also be caused by the different expression of other MADS-box genes in both cauline leaves and flower buds (Figure 5). For instance, SEP genes that play a central role in flower meristem and organ identity, by interacting with
AP1 and among themselves to form multimers during regulation of gene expression [22,39], were highly expressed in AFDL. It has been suggested that redundancy exists for AP1/CAL in specifying floral meristem identity [14]. However, although high expression of $C A L$ in flower buds was observed for AFDL in our study, this could not compensate for the function of AP1 in specifying flower meristem identity and the first- and second-whorl organs, which agrees with the observation that apl mutants show an obvious phenotypic change while cal mutants show only a WT phenotype and apl/cal double mutants result in a complete transformation of floral meristems into inflorescence meristems [11]. CAL may be regulated differently from $A P 1$ because of the distinct expression for these two genes in flower buds of AFDL (Figure 5), and it has been suggested previously that $C A L$ and $A P 1$ are regulated differently by LFY and FT [39]. Additional spatiotemporal analysis of $C A L$ expression in AFDL may shed more light on this issue.

In conclusion, the AFDL strain identified in this study is phenotypically similar to apl mutants with noticeable deviations. The abnormal flower development of AFDL was mainly caused by significantly reduced expression of $A P 1$, but also is attributable to crosstalk among key genes in the regulatory network controlling the transition of vegetative growth to the flowering phase as well as flower development. AFDL exhibits variation in flowering with complex flower structures and merits further molecular characterization to understand better the regulatory molecular network. Therefore, AFDL may serve as a good model to study the complex regulation of flower development, especially interactions of regulatory genes in response to various endogenous signals and environmental cues.

This work was supported by the National Natural Science Foundation of China (30771697), the National Hi-Tech Research and Development Program of China (2006AA10Z122) and the Research Foundation of Jiamusi University (S2010-53).

1 Yant L, Mathieu J, Schmid M. Just say no: Floral repressors help Arabidopsis bide the time. Curr Opin Plant Biol, 2009, 12: 580-586

2 Engelmann K, Purugganan M. The molecular evolutionary ecology of plant development: Flowering time in Arabidopsis thaliana. Adv Bot Res, 2006, 44: 507-526

3 Komeda Y. Genetic regulation of time to flower in Arabidopsis thaliana. Annu Rev Plant Biol, 2004, 55: 521-535

4 Baurle I, Dean C. The timing of developmental transitions in plants. Cell, 2006, 125: 655-664

5 Michaels S D. Flowering time regulation produces much fruit. Curr Opin Plant Biol, 2009, 12: 75-80

6 Borner R, Kampmann G, Chandler J, et al. A MADS domain gene involved in the transition to flowering in Arabidopsis. Plant J, 2000, 24: 591-599

7 Blazquez M A, Weigel D. Integration of floral inductive signals in Arabidopsis. Nature, 2000, 404: 889-892

8 Lee J H, Yoo S J, Park S H, et al. Role of SVP in the control of flowering time by ambient temperature in Arabidopsis. Genes Dev, 2007, 21: 397-402

9 Li D, Liu C, Shen L, et al. A repressor complex governs the integration of flowering signals in Arabidopsis. Dev Cell, 2008, 15: 110-120

10 Irish V F, Sussex I M. Function of the apetala-1 gene during Arabídopsis floral development. Plant Cell, 1990, 2: 741-753

11 Bowman J L, Alvarez J, Weigel D, et al. Control of flower develop- 
ment in Arabidopsis thaliana by APETALAl and interacting genes. Development, 1993, 119: 721-743

12 Irish V F. Patterning the flower. Dev Biol, 1999, 209: 211-220

13 Liu C, Zhou J, Bracha-Drori K, et al. Specification of Arabidopsis floral meristem identity by repression of flowering time genes. Development, 2007, 134: 1901-1910

14 Kempin S A, Savidge B, Yanofsky M F. Molecular basis of the cauliflower phenotype in Arabidopsis. Science, 1995, 267: 522-525

15 Mandel M A, Gustafson-Brown C, Savidge B, et al. Molecular characterization of the Arabidopsis floral homeotic gene APETALA1. Nature, 1992, 360: 273-277

16 Parcy F, Nilsson O, Busch M A, et al. A genetic framework for floral patterning. Nature, 1998, 395: 561-566

17 Wagner D, Sablowski R W M, Meyerowitz E M. Transcriptional activation of APETALA1 by LEAFY. Science, 1999, 285: 582-584

18 Ruiz-Garcia L, Madueno F, Wilkinson M, et al. Different roles of flowering-time genes in the activation of floral initiation genes in Arabidopsis. Plant Cell, 1997, 9: 1921-1934

19 Sundstrom J F, Nakayama N, Glimelius K, et al. Direct regulation of the floral homeotic APETALA1 gene by APETALA3 and PISTILLATA in Arabidopsis. Plant J, 2006, 46: 593-600

20 Pelaz S, Ditta G S, Baumann E, et al. B and C floral organ identity functions require SEPALLATA MADS-box genes. Nature, 2000, 405: 200-203

21 Pelaz S, Gustafson-Brown C, Kohalmi S E, et al. APETALA1 and SEPALLATA3 interact to promote flower development. Plant J, 2001, 26: $385-394$

22 Ditta G, Pinyopich A, Robles P, et al. The SEP4 gene of Arabidopsis thaliana functions in floral organ and meristem identity. Curr Biol, 2004, 14: 1935-1940

23 Karim M R, Hirota A, Kwiatkowska D, et al. A role for Arabidopsis $P U C H I$ in floral meristem identity and bract suppression. Plant Cell, 2009, 21: 1360-1372

24 Hepworth S R, Valverde F, Ravenscroft D, et al. Antagonistic regulation of flowering-time gene SOC1 by CONSTANS and FLC via separate promoter motifs. EMBO J, 2002, 21: 4327-4337

25 Gao D, Zhang H. A new drying method of plant specimens for scanning electron microscopy the t-butyl alcohol freeze-drying method. Acta Bot Sin, 1989, 31: 770-774

26 Gregis V, Sessa A, Colombo L, et al. AGL24, SHORT VEGETATIVE
PHASE, and APETALAI redundantly control AGAMOUS during early stages of fower development in Arabidopsis. Plant Cell, 2006, 18: $1373-1382$

27 Huang D W, Sherman B T, Lempicki R A. Systematic and integrative analysis of large gene lists using DAVID Bioinformatics Resources. Nat Protoc, 2009, 4: 44-57

28 Dennis G J, Sherman B T, Hosack D A, et al. DAVID: Database for annotation, visualization, and integrated discovery. Genome Biol, 2003, 4: P3

29 Shannon S, Meeks-Wagner D R. A mutation in the Arabidopsis TFL1 gene affects inflorescence meristem development. Plant Cell, 1991, 3: 877-892

30 Bradley D, Ratcliffe O, Vincent C, et al. Inflorescence commitment and architecture in Arabidopsis. Science, 1997, 275: 80-83

31 Scortecci K C, Michaels S D, Amasino R M. Identification of a MADS-box gene, FLOWERING LOCUS M, that represses flowering. Plant J, 2001, 26: 229-236

32 Buchovsky A S, Strasser B, Cerdan P D, et al. Suppression of pleiotropic effects of functional CRYPTOCHROME genes by TERMINAL FLOWER 1. Genetics, 2008, 180: 1467-1474

33 Fujiwara S, Oda A, Yoshida R, et al. Circadian clock proteins LHY and CCA1 regulate SVP protein accumulation to control flowering in Arabidopsis. Plant Cell, 2008, 20: 2960-2971

34 Grunewald W, Vanholme B, Pauwels L, et al. Expression of the Arabidopsis jasmonate signalling repressor JAZ1/TIFY10A is stimulated by auxin. EMBO Rep, 2009, 10: 923-928

35 Leibfried A, To J P C, Busch W, et al. WUSCHEL controls meristem function by direct regulation of cytokinin-inducible response regulators. Nature, 2005, 438: 1172-1175

$36 \mathrm{Yu} \mathrm{H}$, Ito T, Zhao Y, et al. Floral homeotic genes are targets of gibberellin signaling in flower development. Proc Natl Acad Sci USA, 2004, 101: 7827-7832

37 Seo E, Lee H, Jeon J, et al. Crosstalk between cold response and flowering in Arabidopsis is mediated through the flowering-time gene SOCl and its upstream negative regulator FLC. Plant Cell, 2009, 21: 3185-3197

38 Raj A, Rifkin S A, Andersen E, et al. Variability in gene expression underlies incomplete penetrance. Nature, 2010, 463: 913-919

39 Parcy F. Flowering: A time for integration. Int J Dev Biol, 2005, 49: 585-593

\section{Supporting Information}

Table S1 Primer pairs used for sequencing the $A P 1$ region

Figure S1 DNA sequence of AP1 and its upstream region in AFDL (Lansberg)

Table S2 Genes differentially expressed (more than two-fold) in flower buds between AFDL and WT plants

Table S3 Genes differentially expressed (more than two-fold) in seedlings of AFDL and WT plants

Table S4 Biological function terms and numbers of genes belonging to groups annotated by DAVID software

Table S5 Differentially expressed genes between AFDL and WT plants responsive to stimuli in both seedlings and flower buds

Table S6 Genes and their primer pairs used for qRT-PCR

Figure S2 Expression of flower regulating genes (in Table S6) in cauline leaves (a,b) and flower buds (c,d) using qRT-PCR

The supporting information is available online at csb.scichina.com and www.springerlink.com. The supporting materials are published as submitted, without typesetting or editing. The responsibility for scientific accuracy and content remains entirely with the authors.

Open Access This article is distributed under the terms of the Creative Commons Attribution License which permits any use, distribution, and reproduction in any medium, provided the original author(s) and source are credited. 